\title{
Mask-assisted electron radiation grafting for localized through-volume modification of porous substrates: influence of electron energy on spatial resolution
}

A. Forner-Cuenca ${ }^{a}$, V. Manzi-Orezzolia , P.M. Kristiansen ${ }^{\text {b,c }}$, L. Gubler ${ }^{a}$, T.J. Schmidt ${ }^{\mathrm{a}, \mathrm{d}}$, P. Boillat $^{\mathrm{a}, \mathrm{e}}$

The spatial resolution aspects of the local modification of porous materials by electron induced graft-polymerization were studied by a combination of experiments and numerical simulations. Using blocking masks, only selected regions of the material were exposed to radiation and subsequently grafted. The main focus of this study is the application to gas diffusion layers, a carbonaceous $200 \mu \mathrm{m}$ thick porous substrate widely used in fuel cells, with the goal of improving water management by locally tuning the wettability. The comparison of experiments performed with different electron energies and 
corresponding simulations shows good agreement, identifying the energy threshold necessary to graft through the material to be approximately $150 \mathrm{keV}$. The impact of electron energy on spatial resolution was studied, showing that the blurring effects due to electron scattering reach a maximum at around $200 \mathrm{keV}$ and are reduced at higher electron energies. Finally, the numerical simulations were used to define the conditions necessary to selectively graft only parts of bilayer fuel cell materials.

Keywords: electron radiation grafting, M onte Carlo simulation, porous material, PEFCs, patterned wettability.

\section{Introduction}

In the last 70 years, the use of electron beam technologies has impacted various fields, such as micro and nanofabrication (Geissler and Xia 2004, Shore 2013, Chen 2015, Padeste and Neuhaus 2015), electron microscopy, curing (Wolff-Fabris et al. 2011, Drobny 2013, Drobny 2013) and welding (Sun and K arppi 1996), among others. Electron-beam lithography (EBL) is a technique based on the use of a focused electron beam to draw custom shapes into socalled resist layers, i.e. an electron-sensitive material, which undergoes either chain scission (polymethyl methacrylate (PM M A)) or cross-linking (e.g. SU-8) upon electron exposure and allows for pattern definition by selective removal of the irradiated (or non-exposed) fraction of the resist upon development. EBL is nowadays used in a number of applications and has been developed over the years to manufacture sub $10 \mathrm{~nm}$ resolution structures (Vieu et al. 2000, A ltissimo 2010). Due to the demanding technology required to focus the beam, EBL is 
a very costly technique (> $2 \mathrm{M} \$ /$ device (Altissimo 2010)). There are, however, other applications that do not require such narrow structures. There have been recent works, mainly in the field of microfluidics, focusing on the production of patterns on a scale of tens to hundreds of micrometers using different technologies, such as plasma (Garrod et al. 2007), UV irradiation (Zahner et al. 2011), among others. To the best of our knowledge, none of these studies have focused on modifications of thick porous materials (thickness $>100 \mu \mathrm{m}$ ) using electron beams, which we address in this work.

Our group has recently developed a method to modify porous gas diffusion layers (GDLs) used in polymer electrolyte fuel cells (PEFCs) (Pasaogullari and Wang 2004, Cindrella et al. 2009). The method is based on electron radiation grafting of the polymeric coating covering the carbon fibers (B oillat et al. 2014, Forner-Cuenca et al. 2015). The grafting polymerization refers to the formation of a copolymer between the original polymer $(\mathrm{pA})$ and a newly added monomer (M) (Stannett 1990, Dargaville et al. 2003, Gubler et al. 2005). The resulting molecular structure can be written as pA-g-pM . Normally, pA is a low cost structural polymer and $\mathrm{M}$ introduces an additional valuable property. In our particular case, $\mathrm{pA}$ is a hydrophobic polymer (fluorinated ethylene propylene copolymer (FEP)) and M is a hydrophilic compound (here acrylic acid) to increase the wettability. Details on the chemical structures and polymerization reactions can be found in our previous work (Forner-Cuenca et al. 2016). Since the minimum expected pattern width to be produced is in the range of $100 \mu \mathrm{m}$, we use masks to block the radiation in the undesired regions. By adequately selecting the electron energy the beam should penetrate the entire material thickness, generating radicals in the polymeric coating located under the mask-free regions. In a subsequent step, the activated materials are brought in contact with a liquid solution containing the hydrophilic monomer and the grafting polymerization starts on the activated regions. The control of the 
experimental conditions (type of monomer, concentration, solvent, temperature, reaction time, etc.) allows for tuning the desired final properties (Forner-Cuenca et al. 2016).

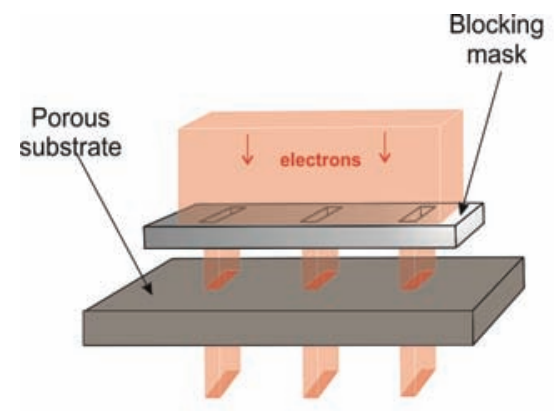

(a) Irradiation

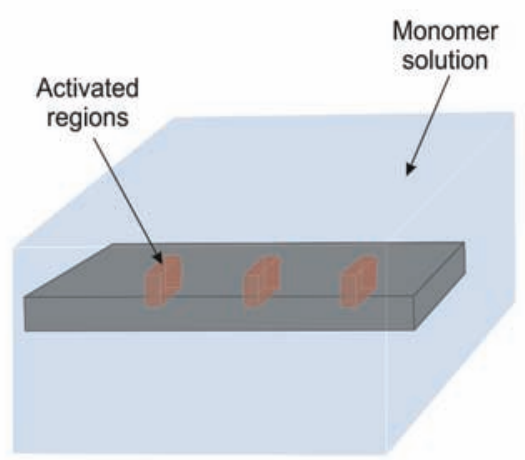

(b) Polymerization reaction

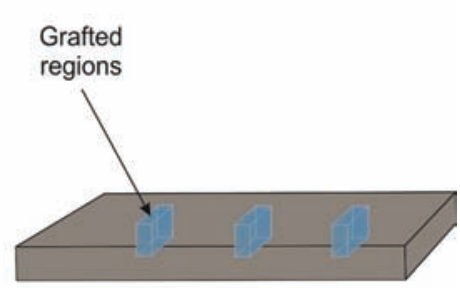

(c) Final material

Figure 1: Illustration of the synthetic process to obtain porous substrates with structured properties. (a) electron radiation using a blocking mask; (b) activated material is brought into monomer solution; (c) finally, a material with localized grafted regions is obtained.

While a number of fields could benefit from the work on modifying thick porous substrates, the implementation of GDLs with patterned wettability in PEFCs is expected to improve the complex water management by providing optimized liquid/gas transport characteristics ( $\mathrm{i}$ and Wei 2009). We have demonstrated significant fuel cell performance increase when using the novel GDLs using the mask-assisted radiation grafting method (Forner-Cuenca et al. 2015, Forner-Cuenca et al. 2016, Forner-Cuenca et al. 2016). The objective of this paper is to provide a more fundamental understanding on how the radiation dose is distributed and, from this knowledge, set the guidelines to produce a second generation of GDLs with patterned wettability with higher resolution and tailored penetration depth.

We start by comparing experimental results of elemental analysis on top and bottom surfaces with M onte Carlo simulations of electron transport in a reproduced geometry. In these lines, we compare the achieved resolution as a function of the electron energy and discuss the 2dimensional dose distribution. A fterwards, the effect of separating the mask and the substrate 
is studied experimentally for different distances. Simulations provide a theoretical basis of the forward scattering phenomena at two energies. Further, we theoretically explored the impact of backscattering by using different materials on the back-side of the substrates. The paper closes by presenting an example of the use of simulations in a predictive way by irradiating a bi-layer material to a pre-selected penetration depth.

\section{Experimental methods and simulations}

The experiments are used to quantify the distribution of grafted molecules on top and bottom surfaces, and the simulations to calculate the absorbed dose distribution through the thickness of the material as well as to extrapolate the results to ranges of energies not accessible with the currently used electron sources. The experimental methodology consists of the following steps: (I) GDLs are electron beam irradiated using masks; (II) activated GDLs react with the monomer, acrylic acid; (III) grafted GDLs are cleaned and exposed to sodium hydroxide $(\mathrm{NaOH})$ in order to replace the protons in the acid groups by a sodium ion $\left(\mathrm{Na}^{+}\right)$for visualization purposes; (IV ) elemental mappings are recorded using energy dispersive X-ray analysis; (V) elemental mappings are processed and profiles of the grafting yield are obtained. The following subsections introduce the materials, procedures and techniques used, alongside with details of the M onte Carlo simulations.

\subsection{M aterials}

\subsubsection{M asks}

$2 \mathrm{~mm}$ thick stainless steel masks were used. Rectangular openings were machined using a pressurized water jet (WATERjet, A arwangen, Switzerland). Two types of masks were used for the studies reported herein: (1) a mask containing rectangular slits of $0.5 \mathrm{~mm}$ width spaced $5 \mathrm{~mm}$; (2) a mask with two perpendicular rectangular slits (500 $\mu \mathrm{m}$ width) forming a cross- 
like shape. The first mask was used for the studies about penetration depth and resolution, while the second mask was used for the beam broadening study to elucidate beam divergences in different spatial directions. The slits of mask (I) were placed in the direction parallel to the linear electron beam axis.

\subsection{2. $G$ as Diffusion Layers (G DL)}

Untreated Toray Paper 060 (TGP-H-060) was used as base GDL material (purchased at Fuel Cell Earth Store). It has a thickness of $\sim 200 \mu \mathrm{m}$, a bulk density of $0.44 \mathrm{~g} \mathrm{~cm}^{-3}$ and a porosity of $\sim 78 \%$. The material was coated with FEP using the "dipping" method followed by a thermal treatment. The details about the coating application were described elsewhere (Forner-Cuenca et al. 2016). For this particular study, a coating load of 70\% (weight of the coating relative to the base material) was used. We decided to use a high coating load to have a sufficient amount of polymer to perform elemental mappings with a good image quality.

\subsubsection{FE P films (as dosimeters)}

Fluorinated ethylene propylene (FEP) films of $25 \mu \mathrm{m}$ thickness were used (DuPont ${ }^{\top M}$ Teflon ${ }^{\circledR}$ FEP Gauge 100) for measuring the beam width in the beam broadening study (Section 3.3). The foils were cut into pieces of $6 \mathrm{~cm} \times 6 \mathrm{~cm}$ and rinsed with abundant ethanol to remove any contaminant present. A fterwards, they were dried at $60^{\circ} \mathrm{C}$ in vacuum overnight before being packed into bags.

\subsection{E lectron beam}

Two electron beam installations covering different energy ranges were used:

1) A linear accelerator was used at LEONI Studer (Däniken, Switzerland) with a variable accelerator energy range of 1.05-2.35 M eV, a beam intensity range of $0-35 \mathrm{~mA}$ and a scan width of $1220 \mathrm{~mm}$. M aterials are mounted on metallic trays that are conveyed through the irradiation facility. Tray speed $[\mathrm{m} / \mathrm{min}]$ and current beam $[\mathrm{mA}]$ are adjusted to control the 
dose. $1 \mathrm{~cm}$ thick polyethylene (PE) was placed between the metallic tray and samples in order to reduce the backscattering. Unless otherwise stated, a fixed electron energy of $2.1 \mathrm{M} \mathrm{eV}$ was used, the other two parameters being variable to achieve the desired dose.

2). Low-energy electron beam treatment was carried out in an EBL ab 200 sealed laboratory emitter system from Comet A G (Flamatt, Switzerland). Samples were subjected to different doses ranging from $50 \mathrm{kGy}$ under nitrogen (<200ppm oxygen), employing an acceleration voltage between 80 and $200 \mathrm{kV}$ and a maximum dose of $50 \mathrm{kGy}$ per pass at a transport speed of $12 \mathrm{~m} / \mathrm{min}$ and a nominal air gap of $10 \mathrm{~mm}$.

Samples and masks were placed on a frame-type holder. This holder was gas sealed by covering the upper part with a $30 \mu \mathrm{m}$ thick polyethylene terephthalate (PET) film and a combination of 0 -rings and screws. The sealing of the holder was done to avoid any decomposition products potentially produced during irradiation to damage the equipment. When specified, $600 \mu \mathrm{m}$ of polyethylene (PE) was placed between the holder and the samples to minimize backscattering.

\section{Grafting reaction}

Polyacrylic acid (pAA) was chosen as grafting system due to the favorable kinetics and easiness to characterize. A crylic acid (Sigma A Idrich 99\% containing 180-200 ppm M EHQ ad inhibitor) was used as received. The monomer was diluted in water down to $15 \%$ by weight. Extensive work was performed to find synthetic conditions allowing a straightforward quantification of the grafting (i.e. a significant amount of acrylic groups leads to color changes and sufficient molecules for the elemental mapping). On the contrary, elevated grafting degrees are known to produce significant expansions of sample size with the FEP-g- 
pAA system. Since the paper discusses localized modifications, we have chosen the conditions resulting in minor size expansion allowing for measureable quantification.

Activated GDLs and FEP films were placed in cylindrical glass reactors (volume around $70 \mathrm{~mL}$ ) together with the grafting solution at atmospheric pressure. In order to de-oxygenate the media, nitrogen flushing of the solution was performed during 60 minutes. A fter this time was completed, the reactors were sealed. Later, the reactors were placed in a controlled temperature water bath at $60^{\circ} \mathrm{C}$ for $30 \mathrm{~min}$. Samples were cleaned using a vacuum filter system (Forner-Cuenca et al. 2015, Forner-Cuenca et al. 2016, Forner-Cuenca et al. 2016) with ethanol, isopropanol and water. Finally, they were dried under vacuum at $60^{\circ} \mathrm{C}$ before further preparation for characterization purposes.

\section{Characterization}

The replacement of protons (from the acidic group) to sodium ions ( $\left.\mathrm{Na}^{+}\right)$to form sodium polyacrylate resulted in a significant improvement of elemental signal measured with energydispersive X-ray analysis (EDX). To do so, samples were punched (6 mm diameter) and immersed into a $0.025 \mathrm{M} \mathrm{NaOH}$ solution (solvent composed of $60 \%$ wt. $\mathrm{H}_{2} \mathrm{O}$ and $40 \%$ wt. EtOH) and stirred overnight. A fter that, they were rinsed with water to remove excess salt and dried under vacuum. 


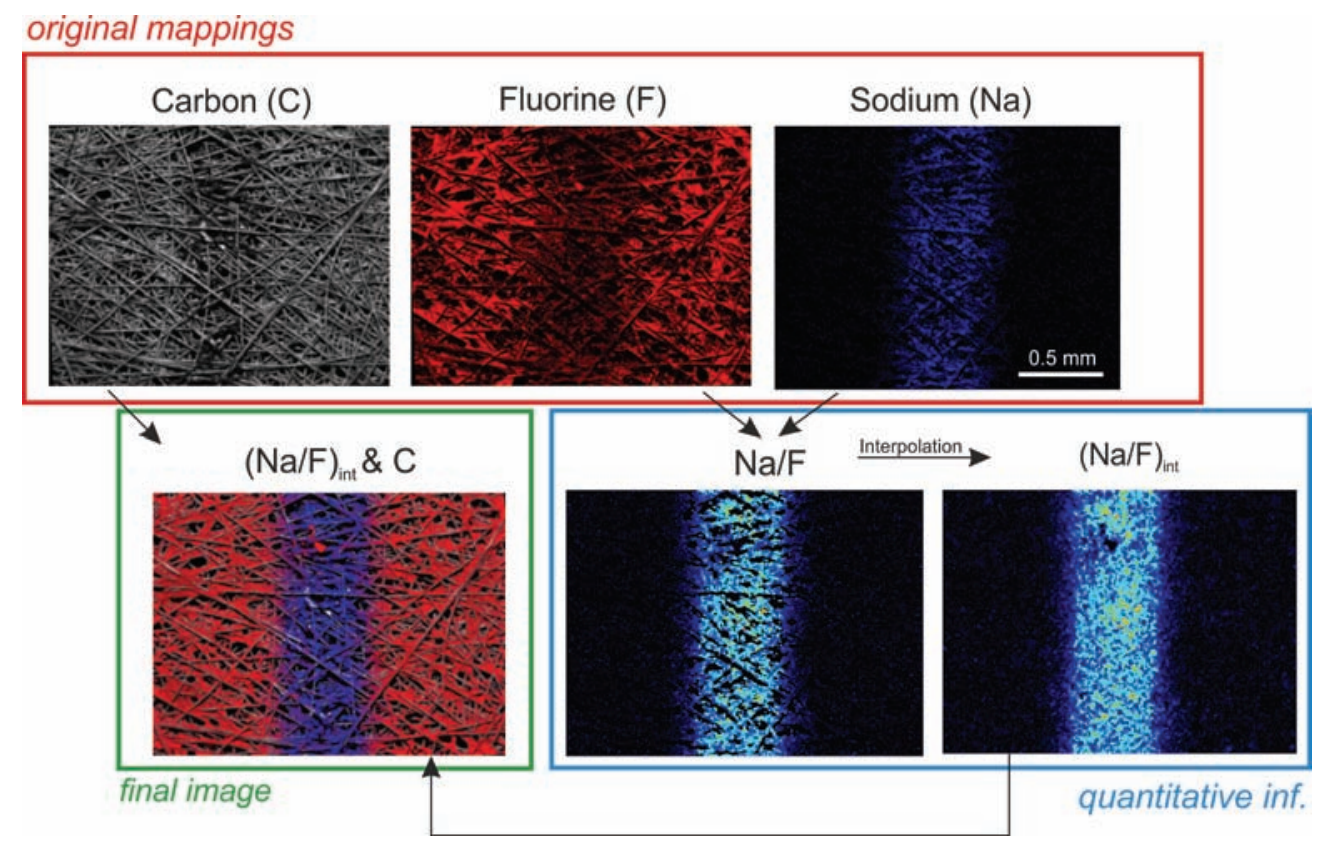

Figure 2: The original EDX mappings for $\mathrm{C}, \mathrm{F}$ and $\mathrm{Na}$ are shown on the top. Processed mappings containing $\mathrm{Na} / \mathrm{F}$, interpolated $\mathrm{Na} / \mathrm{F}$ and a combination of $\mathrm{Na} / \mathrm{F}$ with $\mathrm{C}$ are shown on the bottom. The mappings correspond to a $500 \mu \mathrm{m}$ pattern using $180 \mathrm{keV}$ (on the top surface).

EDX elemental mapping was chosen for the characterization of the patterns. An FE-SEM Ultra 55 (Carl Zeiss, Oberkochen, Germany) with a compatible accessory (EDAX TSL, AMETEK) was used. Elemental mappings were obtained for carbon, fluorine, oxygen and sodium (Figure 2) on the external surfaces of the modified GDL. In this work we refer to "top" as the surface facing the electron beam and "bottom" the opposite surface. The carbon signal comes from carbon fibers and coating (to a lesser extent), while fluorine is exclusively present in the polymeric coating (FEP). Sodium should be exclusively present in the sodium polyacrylic acid molecules, while oxygen comes mainly from the grafted co-polymer and some partially oxidized carbon fibers and FEP. That is the reason why we chose sodium to perform further analysis. It should be emphasized the fact that even if we refer to external surfaces, the EDX does not prove the very external surfaces (like X-ray photoelectron spectroscopy does, for example); however the X-rays are emitted from some deeper locations 
of the material (i.e. 0.5-2 $\mu \mathrm{m}$ ). This effect is shown as "excitation pear". On top of that, the porous and rough nature of the GDL also increases the probability to probe some material layers located underneath. As an arbitrary value, we consider $30 \mu \mathrm{m}$ as the external surfaces within this work.

As shown in Figure 2, we calculated the ratio between $\mathrm{Na}$ and $\mathrm{F}$ signals. Since the coating content and distribution is not the same for each sample, this normalization permits obtaining more reliable results. As the $\mathrm{Na/F}$ ratio as a measure of the amount of grafted polymer only makes sense in locations where the coating is present, a threshold was set on the fluorine (F) signal below which the $\mathrm{Na/F}$ ratio was defined as "unknown". The values in these regions were obtained from the adjacent regions using linear interpolation. Following this, a "final image" was created by overlaying the signals from the fibers $(C)$, the coating $(F)$ and the grafted polymer $(\mathrm{Na} / \mathrm{F})$.

For the measurements of beam broadening using flat FEP films, we used optical microscopy (Leica VZ700 C) and relied on opacity evolvement in the modified areas. The originally transparent FEP film becomes opaque in the regions where a significant amount of polyacrylic acid has been grafted (FEP-g-pAA). It must be noted that very small grafting degrees may not lead to visually identifiable loss of transparency. For this reason, the results obtained with this method are semi-quantitative.

\subsection{Simulations}

\subsubsection{E nvironment}

The code system PENELOPE (Penetration and Energy Loss of Positrons and Electrons) was used for performing the simulations presented in this paper (version 2015) (Salvat F. , Sempau et al. 1997, Sempau et al. 2003, Francesc and J osé 2009). PE NEL OPE performs M onte Carlo 
simulations of coupled electron-photon transport in arbitrary materials for a wide energy range (50 eV - $1 \mathrm{GeV}$ ). The code system contains state-of-the-art physics for particle transport. We prepared scripts to simulate the various geometries and source characteristics described in the respective chapters. If not otherwise stated, $10^{7}$ electrons were used for each of the simulations.

\subsubsection{G eometry definition}

In order to represent the studied system, a geometry space was defined as shown in Figure 3. Generally speaking, it consists of a stack of horizontal planes which are infinite in the y direction (perpendicular to the paper) and infinitively long in the $x$ direction. The electron beam source has been placed above the Body I at a distance of $10 \mathrm{~mm}$. We have considered mono-directional beam flux as a good representation of the experimental situation where a frame moves and gets irradiated using a linear source. We considered the source to be sufficiently long in $\mathrm{x}$ and $\mathrm{y}$ direction and $5 \mathrm{~mm}$ in $\mathrm{z}$ direction. B ody I corresponds to a layer of PET, used to seal the irradiation frame (as described in 2.2). This layer is $\sim 30 \mu \mathrm{m}$ thick and has been included in the simulations. B ody II corresponds to the steel mask. It is $2 \mathrm{~mm}$ thick and has a slit opening of $0.5 \mathrm{~mm}$. Body III represents the material of interest (GDL) and is $200 \mu \mathrm{m}$ thick. Normally, the distance between the mask and the GDL ( $\left.D_{\mathrm{ms}}\right)$ is $0 \mu \mathrm{m}$, except in the study about the impact of mask-GDL distance (Section 3.3). Body IV is only considered in some cases and can refer to an additional layer with different properties (such as microporous layer) or to a material underneath to modify the backscattering. In the case that an MPL is considered, the distance between Body III and Body IV $\left(D_{s b}\right)$ is $0 \mu \mathrm{m}$, however two distances were used ( 0 and $200 \mu \mathrm{m}$ ) when a backscattering material was considered. 


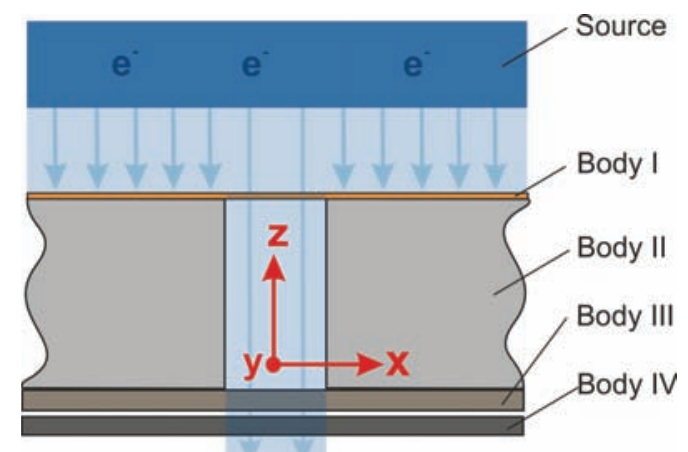

Figure 3: Illustration of the geometry considered (not on scale) for performing the simulations.

\subsubsection{M aterial properties}

Table 1 contains a collection of the materials used in this work and the most relevant properties. Some materials were already included in the PENELOPE database and therefore no elemental composition needed to be input. The steel elemental composition used is obtained from a typical stainless steel $316 \mathrm{~L}$ type as used in the experiments. The elemental composition for GDL and M PL was calculated considering the FEP polymer content ( $70 \%$ wt. in the GDL and $30 \%$ in the MPL) and the material porosity ( 0.70 for the GDL and 0.24 in the MPL). The porous material was represented by using an apparent density computed by the contributions of solid materials and air void. Future work will include the utilization of complex geometries to capture the effect of different fiber orientations.

Table 1: List of materials used in this work and their relevant characteristics.

\begin{tabular}{cccc}
\hline Name & Thickness $[\mathbf{\mu m}]$ & Density $\left[\mathbf{g ~ c m}^{-3}\right]$ & Composition \% wt. \\
\hline PET (Body I) & 0.03 & 1.40 & - \\
Steel (B ody II) & 2.0 & 7.90 & 17(Cr), 12(Ni), 81(Fe) \\
GDL (B ody III) & 0.2 & 0.75 (Rashapov et & $31(\mathrm{~F}), 69(\mathrm{C})$ \\
FEP (Body III) & 0.2 & al. 2015) & -
\end{tabular}




$\begin{array}{cccc}\text { M PL (Body IV) } & 0.05 & \text { Newman 2005) } & \text { 18(F), 82(C) } \\ \text { PE (Body IV) } & 0.10 & 0.940 & -\end{array}$

\section{Results and Discussion}

\subsection{Penetration depth and resolution dependence on electron energy}

The first section of results focuses on the effects of electron energy on the resolution and penetration depth. We start by showing the experimental results of the EDX mapping at varying electron energies (Figure 4). For every energy value, it can be clearly seen that a sharply defined pattern of around $500 \mu \mathrm{m}$ appears on the top surface. M ore details on dose distribution will be discussed in Figure 5 and following paragraphs. A nalyzing the bottom surfaces, no measurable grafted pattern was observed on samples irradiated with the lowest energies (120 and $140 \mathrm{keV}$ ), which indicates that electrons are not penetrating the complete material thickness, or that the dose absorbed at the bottom surface in too low to allow measurable grafting polymerization. 


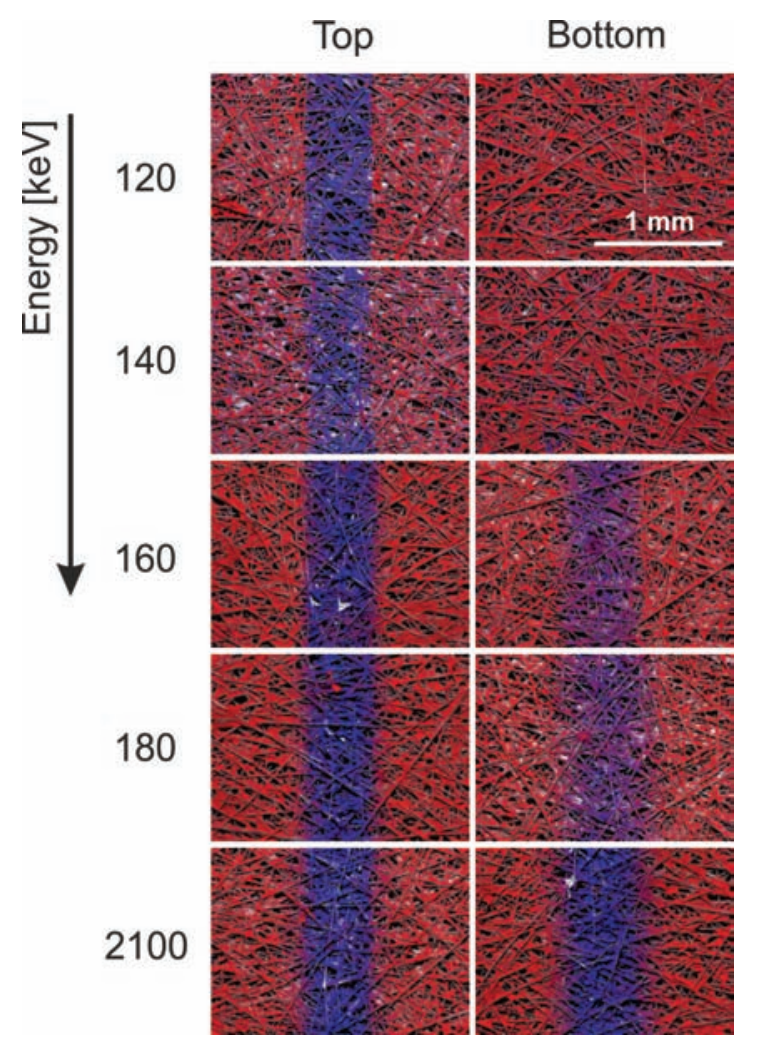

Figure 4: Processed EDX mappings at different electron energy values and showing the top and bottom external surfaces of the G DL. Similar to Figure 2, red and blue color represents F and $\mathrm{Na} / \mathrm{F}$, respectively.

At higher energies, $\mathrm{E}>160 \mathrm{keV}$, the pattern also appears at the bottom surface and has a similar width as the pattern at the top. However, the relative concentration of grafted molecules seems to be lower, which could indicate a reduced dose reaching the bottom surface or a less effective grafting processing occurring there, the second option being less probable considering the reactor design (Forner-Cuenca et al. 2016) . Interestingly, similar grafting intensity is found at the top and bottom surfaces of the modified GDL irradiated with the highest energy $(2100 \mathrm{keV})$. This observation points to a more homogeneous distribution of the absorbed dose and effective grafting occurring on both sides. It should be noted that the pattern obtained at the bottom surface is slightly more blurred than the one at the top surface. Figure 5 shows simulated cross-sectional 2-dimensional dose distribution images. It can be seen that maximum dose values are obtained in the center while the broadening and blurring 
due to scattering events appear to become significant in the energy range $E=140-200 \mathrm{keV}$. A region having a width of approximately $100 \mu \mathrm{m}$ adjacent to the area where grafting is desired is thus exposed to radiation as well. The dose distribution over the $x-z$ directions follows a conical shape, which broadens towards the bottom surface, thereby impairing the resolution at the bottom surface compared to the one at the top surface.

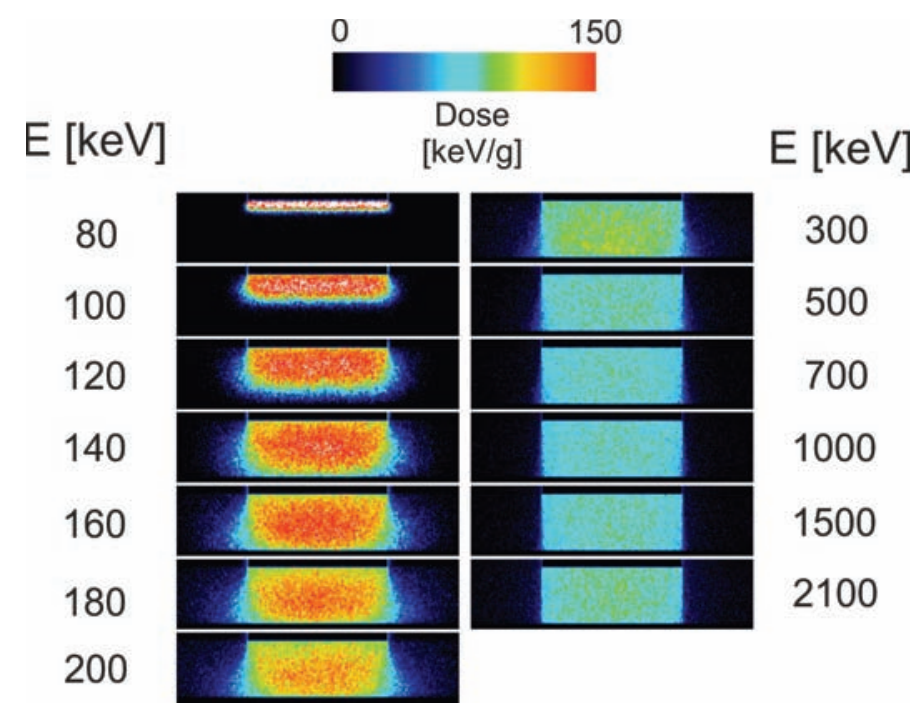

Figure 5: Simulation results of 2D dose distribution at the GDL for different values of electron energy.

Surprisingly, this effect is predicted to notably disappear when increasing the energy from 200 $\mathrm{keV}$ to $300 \mathrm{keV}$. In this case, the dose distribution becomes more homogeneous and scattering is significantly reduced, thereby defining higher quality patterns. This trend persists at elevated energies and similar results are obtained in the energy range between 500-2100 keV. It should be noted that a slightly higher residual dose appears on the mask covered regions at the highest energy, $\mathrm{E}=2100 \mathrm{keV}$, which could originate from secondary electrons and B remsstrahlung transported throughout the mask.

In order to assess the resolution obtained on top and bottom surfaces, we systematically calculate the $\mathrm{Na} / \mathrm{F}$ intensity profiles at different energy values and display them alongside the 
simulation results of dose absorbed (Figure 6). The dose profiles were calculated considering the outer $30 \mu \mathrm{m}$ and averaging over the $z$ direction. From experiments, it can be seen that electron radiation does not penetrate throughout the complete material at $\mathrm{E} \leq 140 \mathrm{keV}$ (Figure $6 a$ and $6 b)$. Differences between experimental values and simulations are notable, especially in the case of $140 \mathrm{keV}$. The explanation for this could be the real PET layer thickness. If the layer would be slightly thicker (i.e. 5-10 $\mu \mathrm{m}$ ) this would significantly impact penetration depth of the porous material underneath. The definition of the porous material used in this work can also impact the results. Finally, also differences in the real air gap may have an impact despite to a lesser extent than the above.
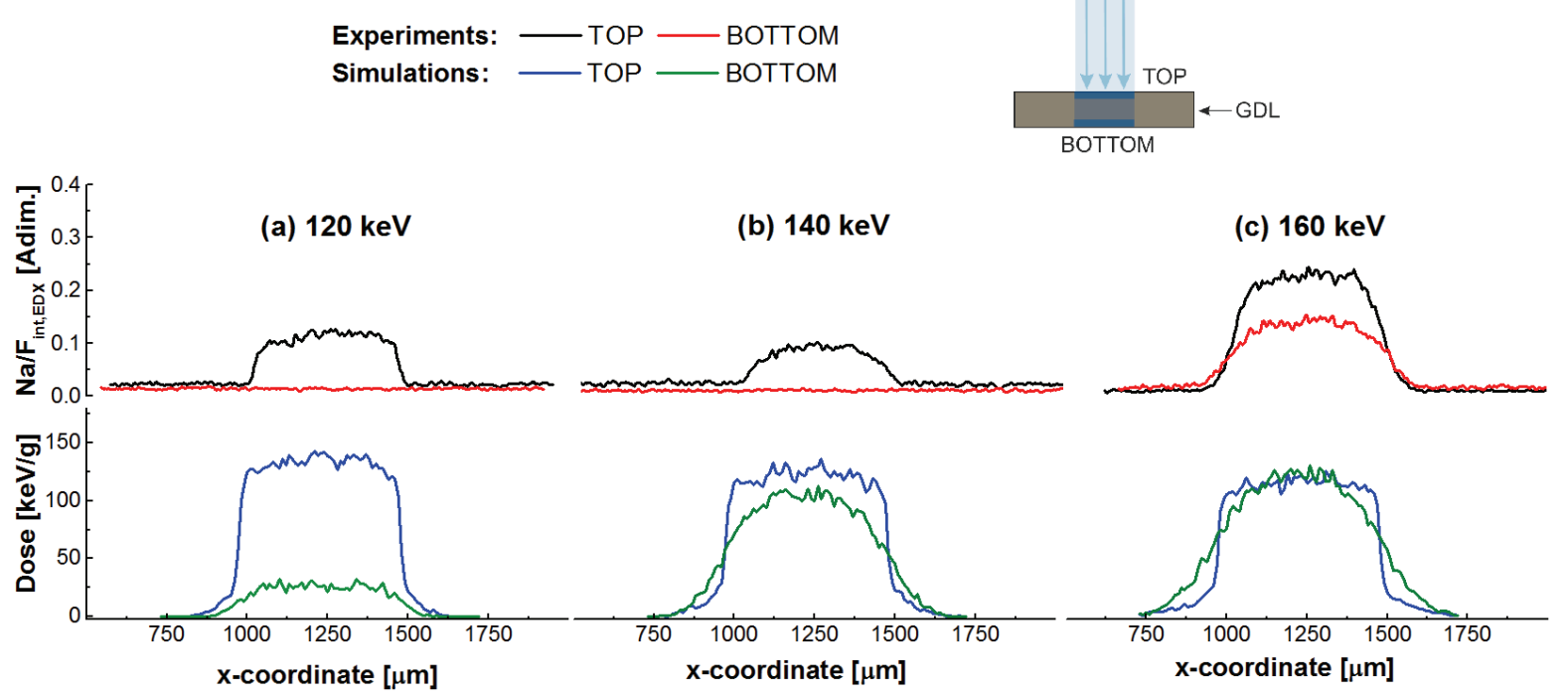

(d) $180 \mathrm{keV}$

(e) $2100 \mathrm{keV}$
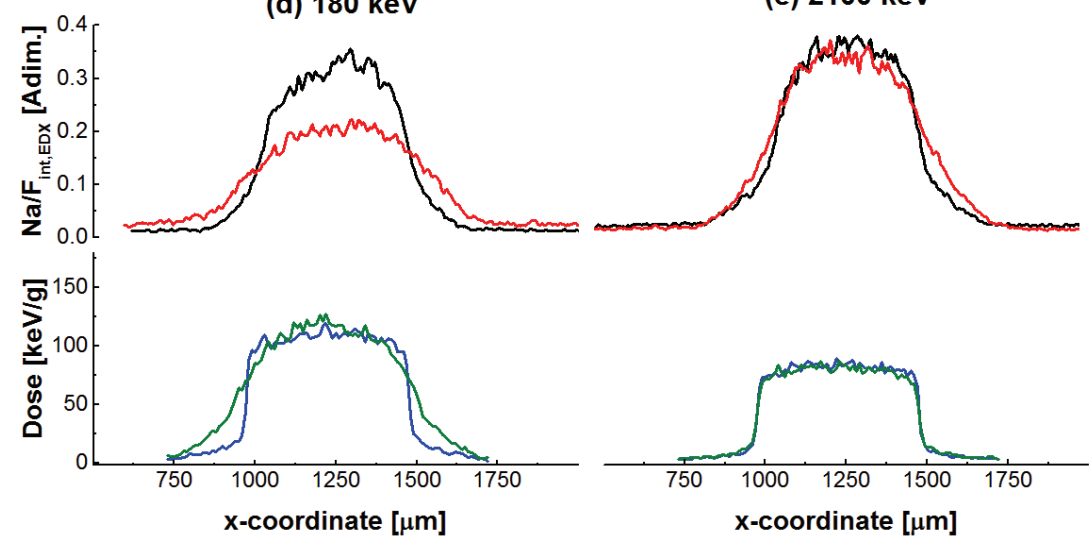
Figure 6: Comparison of experimental and simulation results top and bottom of the GDL.

Let us compare the experimentally obtained profiles at different energies for the top surface. A trend shows broadening of the modified regions as energy increases. The simulation results capture this trend and fairly similar pattern widths and sharpness are obtained for every value of energy. We speculate that these differences appear due to deviations of beam monodirectionality. If the electron flux does not impact the mask perpendicularly, then electrons travelling with angular trajectories have higher probabilities to be stopped by the mask side walls before reaching the GDL if they have a lower energy. This phenomenon would also justify the blurring of the edges of the modified domain, contrary to simulations that show a sharp transition. On the bottom surfaces, a broadening and blurring of the modified domain is observed as well, with a larger magnitude than for the top surface for the energies lower or equal to $180 \mathrm{keV}$ (Figure $6 \mathrm{c}$ and $6 \mathrm{~d}$ ). This corresponds well to the simulation results predicting a difference in width and sharpness between the top and bottom surfaces. At the highest energy $(E=2100 \mathrm{keV})$, the top and bottom surface have nearly the same pattern width and sharpness, which is well represented by the simulation results. A slightly broader pattern is obtained at the bottom interface. This can be explained by backscattering originating from the holder materials underneath and will be discussed in further detail in Section 3.3.

It is important to stress the fact that $\mathrm{Na} / \mathrm{F}$ intensity values obtained with the EDX are not linearly proportional to the dose calculated with the simulations. Several reasons contribute to such differences: (a) the relationship between dose and degree of grafting is not linear and complexly depends on monomer type, concentration, temperature, reaction time, etc.; (b) Xrays detected in EDX are not emitted only from the very external surfaces, but from a volume of some hundreds of nanometers depth (effect known as "ionization pear"); (c) the porous and 
heterogeneous nature of the GDL material can impair the quantitativeness of the analysis due to, for instance, incoming emitted $X$-rays from lower material layers. Consequently, one should bear in mind that EDX profiles at best provide a semi-quantitative description of the measurable grafting locations. In this work we do not intend to use the Monte Carlo simulations to reproduce in detail the experimental observations, but rather to provide a fundamental understanding of electron transport and energy absorbed in localized regions of thick materials. This will allow us to define irradiation strategies and design experimental setups to modify thick porous materials with improved pattern quality.

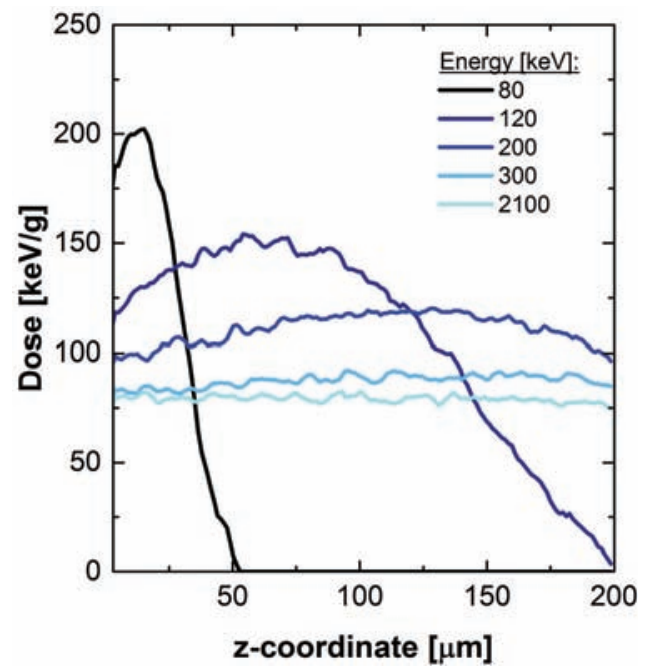

Figure 7: Simulation results of absorbed dose over the z-coordinate (in the GDL) at five different energies.

The current analysis procedure does no give experimental access to the depth profile of the material modification, thus this aspect will be analyzed on the basis of simulation results. The experimental measurements on the top and bottom surfaces serve as a validation of these simulations, taking into account the slight discrepancy observed in the energy threshold necessary to penetrate through the material. Figure 7 shows the distribution of the absorbed dose over the GDL thickness according to the simulation. The maximum absorbed dose 
displaces to deeper z-coordinates as energy increases. Interestingly, for $E=300 \mathrm{keV}$, the dose distribution on the GDL is rather homogeneous, and there are only marginal changes when increasing this energy up to $2100 \mathrm{keV}$. The depth profile information is interesting for the practical application to fuel cells for the following reasons: (a) to define the minimum energy required in case the same properties throughout the complete material thickness are desired; (b) to pre-design modifications with selected penetration depths.

\subsection{Influence of the spacing between mask and sample}

The distance between the blocking masks and the irradiated substrate, $D_{m s}$, has a notable influence in defining the pattern of the targeted width. Figure 8 shows the beam broadening as a function of $D_{\mathrm{ms}}$. B eam broadening, $B B$, has been calculated as:

where $w_{f}$ is the final pattern width after reaction and $w_{0}$ is the original mask opening width $(500 \mu \mathrm{m})$. In this study, we differentiate between the direction parallel to the electron beam scanning direction, $\Delta \mathrm{x}$, and the direction perpendicular to the beam axis, $\Delta y$ (Figure 8a). At the lowest energy (Figure 8b), E = $200 \mathrm{keV}$, it can be seen that significant broadening occurs ( 2.3 $\mathrm{mm}$ when the separation in $10 \mathrm{~mm}$ ). There is a fairly good linear fit with a slope of $\sim 0.23 \mathrm{~mm} / \mathrm{mm}$ for both directions ( $\Delta \mathrm{x}$ and $\Delta \mathrm{y}$ ). This could be explained by the geometry of the electron source and the position of the sample with respect to it. At higher energies (Figure 8c), however, significantly different beam broadening occurs in the two directions. The broadening in the $\Delta x$ direction (slope $\sim 0.35 \mathrm{~mm} / \mathrm{mm}$ ) amounts by a factor greater than two compared to that in the perpendicular direction, $\Delta y$ (slope $\sim 0.16 \mathrm{~mm} / \mathrm{mm}$ ). The electron beam used at LEONI sweeps a $30^{\circ}$ angle between the linear source and the actual sample location. It can be expected that electrons hitting the mask near the slit oriented in the $\Delta x$ 
direction will follow trajectories deviating from the main axis, which leads to more pronounced scattering events and loss of resolution.

(a)
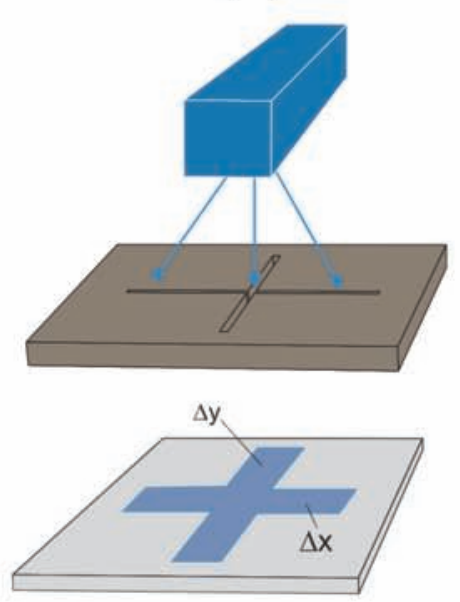

(b)
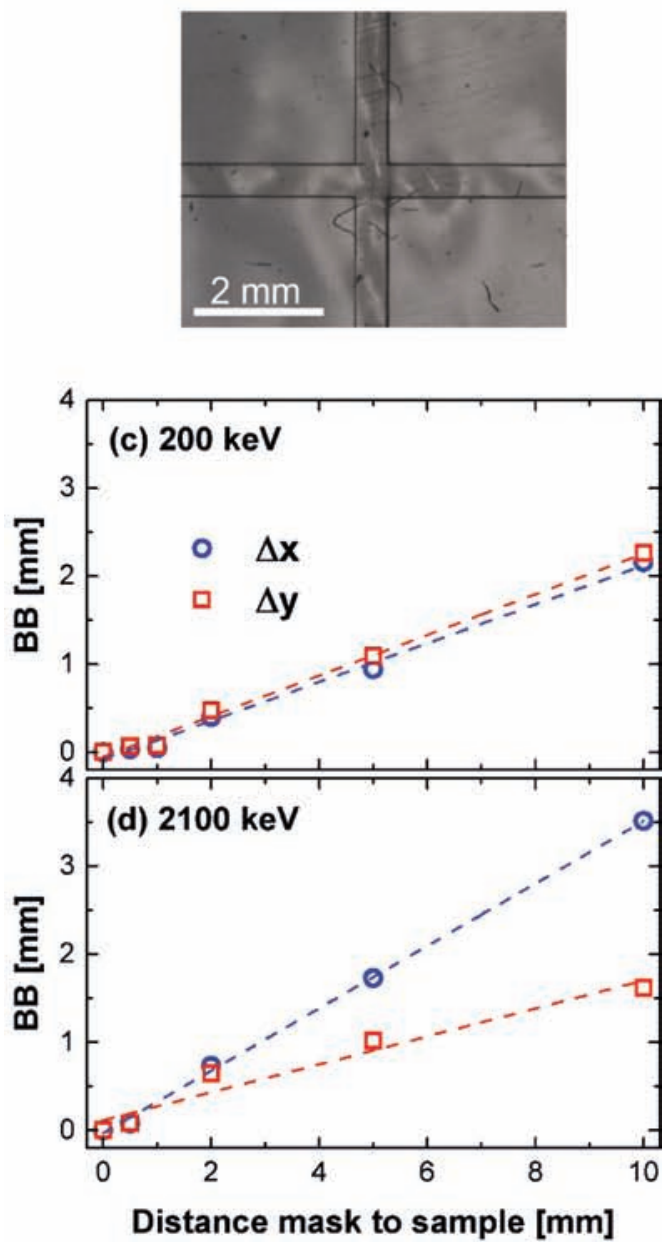
Figure 8: B ream broadening versus mask-to-sample distance for the two spatial directions at two electron energies: (a) Illustration of the setup used and definition of directions; (b) optical micrograph showing the grafted structure; (c) $200 \mathrm{keV;}$ (d) $2100 \mathrm{keV}$.

The experimental results previously discussed (Figure 8) are highly dependent on the type of electron beam (geometry, source type, dose rate, etc.) and it is therefore difficult to extract general conclusions. In order to provide a more fundamental description of the phenomena, we performed M onte Carlo simulations considering an idealized geometry as shown in Figure 9a. Since we assume a mono-directional electron beam, the objective of this study is to quantify the resolution loss only due to small angle scattering events as function of the distance between a mask and the sample of interest. FEP flat thin films were used to simplify the problem as compared to thick porous materials and to reproduce the experiments. At $\mathrm{E}=$ $200 \mathrm{keV}$ (Figure 9b), it can be seen that very significant scattering occurs as $D_{m s}$ increases and the dose distributes over the adjacent regions. Interestingly, at $E=2100 \mathrm{keV}$, this effect is nearly eliminated and only slight differences exist on the dose distributed at various values of $D_{m s}$, which points to a strong influence of energy in these scattering events. For the low energies $(<200 \mathrm{keV})$, the effect of scattering according the simulation is in the same order of magnitude as the experimentally observed broadening. At high energy (2100 keV), the observed broadening can hardly be explained by the effect of scattering, in particular for the $\Delta x$ direction, and the observed effects are rather imputable to the angle of the electron beam, as mentioned above. 
(a)
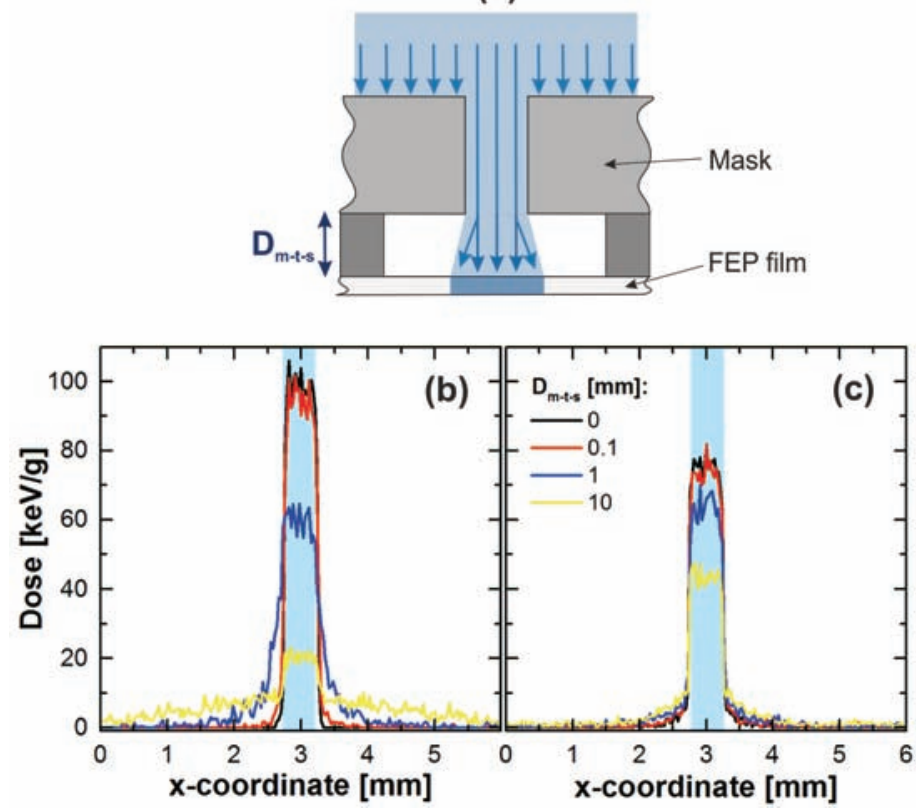

Figure 9: Simulation results of beam broadening as a function of the distance between mask and sample. (a) Schematic of the configuration used for simulation; (b, c) Dose distribution over $x$-coordinate at (b) $200 \mathrm{keV}$ and (c) $2100 \mathrm{keV}$, respectively.

\subsection{Backscattering as a limitation for achievable resolution}

A n important consideration when targeting high definition modifications on both sides of the porous material is the backscattering (i.e. large angle scattering events). In this work, we refer to backscattering as radiation that is emitted back to the body of interest after reaching some materials underneath. Depending on the used setup, these could be holders (metallic or polymeric), air or vacuum. In previous experiments of our group, we have experienced that the use of high energy electron beams (i.e. $E=2100 \mathrm{keV}$ ) combined with metallic holding trays lead to poor quality of modifications. In other words: while the directly exposed regions appeared highly activated as desired, the mask-covered areas are still significantly modified. This leads to a final material of poor quality. In our particular case, the complete material would become hydrophilic. To avoid this important issue, we tested different materials as support for the mask-GDL arrangement and found that polyethylene (PE) effectively reduced 
the backscattering effect and allowed the production of materials with a well-defined spatial definition of the desired modification (i.e. containing hydrophilic and unmodified hydrophobic regions). For the sake of providing some fundamental explanations to these observations, we performed simulations comparing the effects of using different materials underneath the GDL (Figure 10a). Due to its practical relevance, the effect of the distance between the GDL and this material is also evaluated $\left(D_{s b}\right)$.

(a)

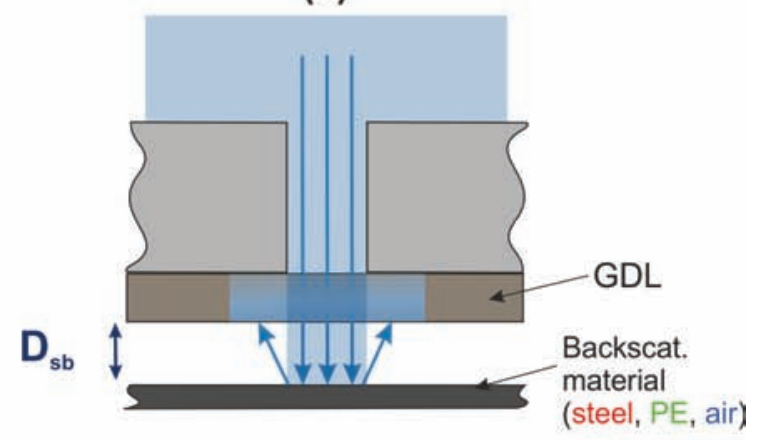

$0 \quad 100$

(b) Steel

Dose

(c) Air
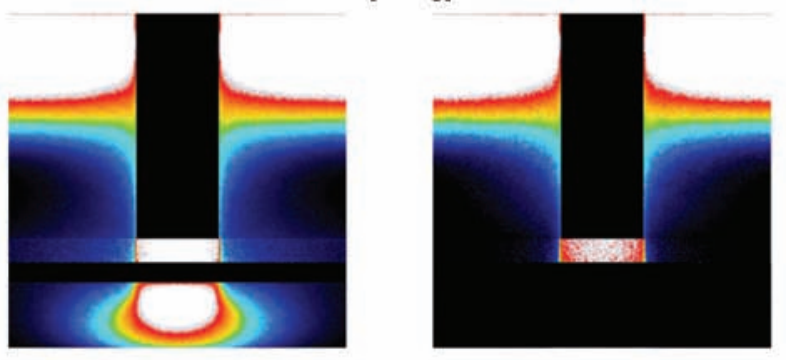

(d) $D_{\mathrm{sb}}=200 \mu \mathrm{m}$

(e) $D_{\text {sb }}=0 \mu \mathrm{m}$

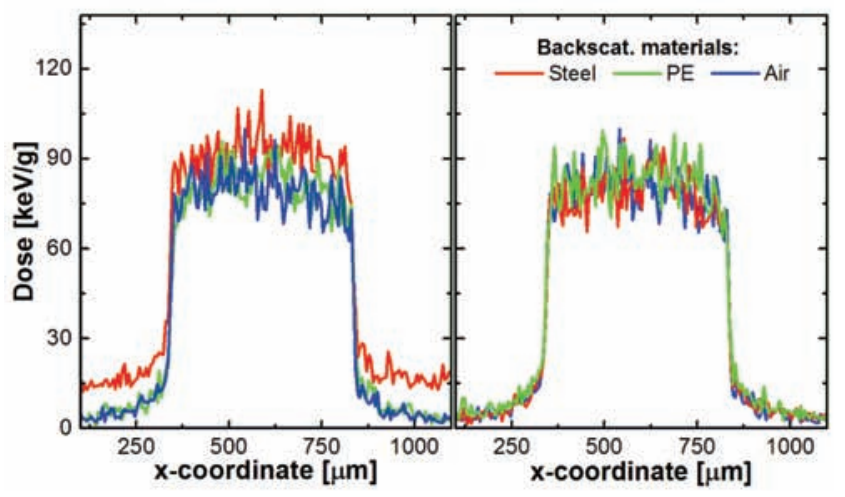

Figure 10: Simulation results on backscattering. (a) Schematic of the configuration used for simulation; 2D dose distribution using (b) steel and (c) air; Dose distribution over x-coordinate at (d) $D_{\mathrm{sb}}=\mathbf{2 0 0} \mu \mathrm{m}$ and 
(e) $D_{s b}=0 \mathrm{~mm}$. These calculations were carried out with $10^{8}$ electrons and an electron energy of $E=2100$

keV.

Very interestingly, it can be seen that the use of steel instead of PE or air leads to a significantly higher dose absorbed in the adjacent regions when using a $D_{s b}=200 \mu \mathrm{m}$ (Figure 10b). This effect essentially disappears when putting the GDL and the backscattering material in direct contact $\left(D_{s b}=0\right)$, which indicates that a minimum spacing is needed to facilitate the electron trajectory after the large angle scattering event. The use of PE instead of having a large space filled with air behind the sample does not result in a visible change of the dose absorbed by the GDL sample. This is consistent with the literature, as it is well known that high atomic number elements - which are absent in PE - favor the backscattering (Rooks).

\subsection{A pplication to bi-layer materials}

Based on the model validation with our experimental observations, we are in a position to use the Monte Carlo simulations in a predictive way. When producing material modifications using the mask-assisted electron beam induced grafting approach, it is important to have the possibility to control the penetration depth to, for example, irradiate one layer only without imparting significant radiation to the layer underneath. State-of-the-art fuel cell diffusion layers are bi-layer materials, composed of a fibrous substrate layer (carbon paper or felt) and a so called micro-porous layer (MPL). This material, composed of carbon particles and polytetrafluoroethylene (PTFE), has a much finer porosity $(\sim 0.1 \mu \mathrm{m}$ pore size) than the substrate and is meant to improve the contact to the catalyst as well as the water management. Depending on the design, a modification of the complete thickness or of only one of these layers - typically the substrate layer - may be desired. 
(a)

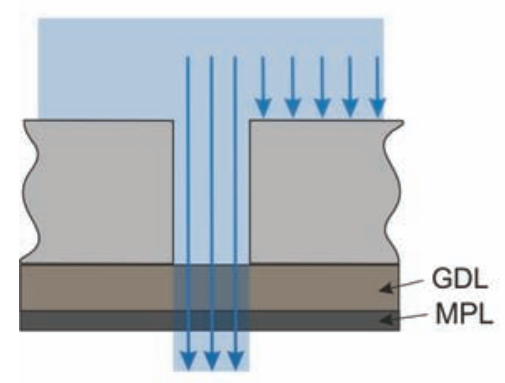

(b)

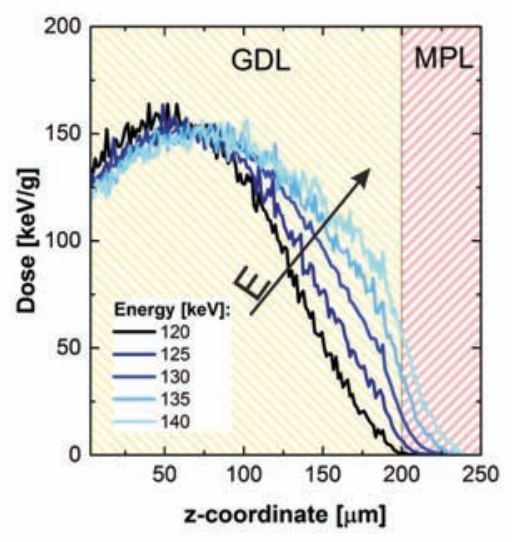

(c)
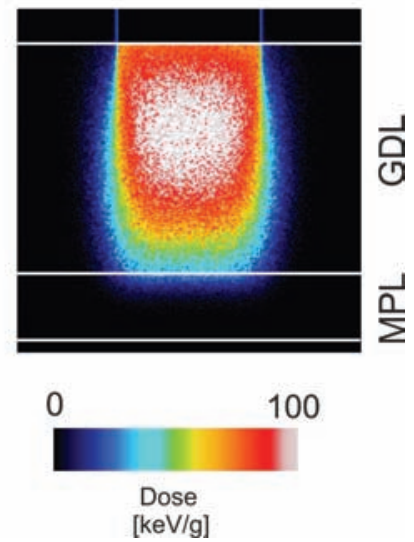

Figure 11: Simulation results on pre-selected penetration depth of a bi-layer arrangement G DL +M PL . (a) Schematic of the configuration used for simulation; (b) D ose distribution over z-coordinate at electron energies of 120, 125, 130, 135 and $140 \mathrm{keV}$; (c) 2D dose distribution at E = $130 \mathrm{keV}$ where the $z$ direction has been stretched by a factor of 4 .

In order to predict the required energy to modify only the substrate in a bilayer arrangement (Figure 11a), we ran simulations (energy range between 120-140 keV) on a sample composed of two layers (each of them with their corresponding parameters, see Experimental section). As seen in Figure 10b, there needs to be a trade-off between having a sufficient dose on the bottom GDL side and minimal damage on the MPL side. We selected $E=130 \mathrm{keV}$ as the most suitable electron energy to meet these requirements and computed the 2-D dose distribution on the stack of materials.

\section{Conclusions}

A combined experimental and numerical analysis based on M onte Carlo simulations was conducted to evaluate the requirements on electron beam irradiation to perform a local modification of porous materials - in particular for fuel cells - by radiation grafting. The major findings and recommendations are: 
- Electron energies larger than $140 \mathrm{keV}$ are needed to modify $200 \mu \mathrm{m}$ thick porous gas diffusion layers throughout their thickness. These results are supported by Monte Carlo simulations on electron scattering in low density carbon coated materials, though the simulation predict a slightly lower threshold energy.

- The edges of the modified regions are increasingly blurred with increasing energies until a maximum is reached at around $180-200 \mathrm{keV}$. Simulations predict that this blurring is significantly reduced again when increasing the energy to $300 \mathrm{keV}$. The experiments performed at much higher energies (2100 keV) partly confirm this trend. These results identify the energy of $300 \mathrm{keV}$ as an optimal trade-off between the need of a sharp pattern and a minimal mask thickness.

- The distance between the blocking mask and the irradiated sample plays an important role. Small angle scattering events, phenomena known as forward scattering, impair the resolution significantly. Reduced energies $(E=200 \mathrm{keV})$ lead to significantly higher beam broadening compared to higher energies. When using lower energies, samples should be placed very near $(<1 \mathrm{~mm})$ to the blocking mask while this is substantially less relevant at higher energies - provided the beam source is arranged perpendicular to the sample in terms of electron flux direction.

- The use of low atomic number compounds at the bottom of irradiated materials reduces significantly the backscattered electrons, thereby improving the quality of the achievable spatial pattern definition. To avoid backscattering, we propose to free the bottom interface if possible for irradiation. When practical reasons due not permit so, polyethylene should be use to minimize the backscattering. 
- The simulations of electron penetration depth indicate the possibility of performing a selective modification of only a selected substrate layer in state-of-the-art bi-layer fuel cell materials.

\section{Acknowledgments}

The authors gratefully acknowledge the Swiss National Science Foundation (SNSF) for funding (project no. 143432). The Swiss Competence Center for Energy Research (SCCER): Efficiency in Mobility is acknowledged for supporting this work.

\section{References}

Product data sheet, Stainless Steel 316/316L AKA Steel, http://www,aksteel.com/pdf/ markets_products/stainless/austenitic/316 316I_data_sheet.pdf, accessed date: 1.06.2016.

Altissimo, M. (2010). "E-beam lithography for micro-nanofabrication." Biomicrofluidics 4(2): 026503.

Boillat, P., Forner-Cuenca, A., Gubler, L., Padeste, C. and Büchi, F. N. (2014). "EP14184065.2." EP14184065.2.

Chen, Y. (2015). "Nanofabrication by electron beam lithography and its applications: A review." Microelectronic Engineering 135: 57-72.

Cindrella, L., Kannan, A. M., Lin, J. F., Saminathan, K., Ho, Y., Lin, C. W. and Wertz, J. (2009). "Gas diffusion layer for proton exchange membrane fuel cells-A review." Lournal of Power Sources 194(1): 146-160.

Dargaville, T. R., George, G. A., Hill, D. J. T. and Whittaker, A. K. (2003). "High energy radiation grafting of fluoropolymers." Progress in Polymer Science 28(9): 1355-1376.

Drobny, J. G. (2013). 5 - Electron B eam Processing of Commercial Polymers, M onomers, and Oligomers. Ionizing Radiation and Polymers, William A ndrew Publishing: 101-147.

Drobny, J. G. (2013). 6 - Industrial A pplications of I onizing Radiation. Ionizing Radiation and Polymers, William A ndrew Publishing: 149-212. 
Forner-Cuenca, A., Biesdorf, J., Gubler, L., K ristiansen, P. M ., Schmidt, T. J. and B oillat, P. (2015). "Engineered Water Highways in Fuel Cells: Radiation Grafting of Gas Diffusion Layers." A dvanced M aterials 27(41): 6317-6322.

Forner-Cuenca, A., Biesdorf, J., Lamibrac, A., M anzi-Orezzoli, V., Büchi, F. N., Gubler, L., Schmidt, T. J. and Boillat, P. (2016). "Advanced Water Management in PEFCs: Diffusion Layers with Patterned Wettability: II. M easurement of Capillary Pressure Characteristic with Neutron and Synchrotron Imaging." Lournal of The Electrochemical Society 163(9): F1038F1048.

Forner-Cuenca, A ., B iesdorf, J., M anzi-Orezzoli, V., Gubler, L., Schmidt, T. J. and B oillat, P. (2016). "Advanced Water Management in PEFCs: Diffusion Layers with Patterned Wettability: III. Operando characterization with neutron imaging." Lournal of The Electrochemical Society 163(13): F 1389-F1398.

Forner-Cuenca, A., M anzi-Orezzoli, V., Biesdorf, J., Kazzi, M. E., Streich, D., Gubler, L., Schmidt, T. J. and Boillat, P. (2016). "Advanced Water Management in PEFCs: Diffusion Layers with Patterned Wettability: I. Synthetic Routes, Wettability Tuning and Thermal Stability." Lournal of The Electrochemical Society 163(8): F 788-F801.

Francesc, S. and J osé, M. F.-V. (2009). "Overview of physical interaction models for photon and el ectron transport used in M onte Carlo codes." M etrologia 462): S112.

Garrod, R. P., Harris, L. G., Schofield, W. C. E., McGettrick, J., Ward, L. J., Teare, D. O. H. and Badyal, J. P. S. (2007). "M imicking a Stenocara Beetle's Back for M icrocondensation Using Plasmachemical Patterned Superhydrophobic-Superhydrophilic Surfaces." Langmuir 23(2): 689-693.

Geissler, M. and Xia, Y. (2004). "Patterning: Principles and Some New Developments." Advanced M aterials 16(15): 1249-1269.

Gubler, L., Gürsel, S. A. and Scherer, G. G. (2005). "Radiation Grafted M embranes for Polymer Electrolyte Fuel Cells." Fuel Cells 5(3): 317-335.

Ji, M. and Wei, Z. (2009). "A Review of Water Management in Polymer Electrolyte M embrane Fuel Cells." Energies 2(4): 1057.

Padeste, C. and Neuhaus, S. (2015). Polymer M icro- and Nanografting, Elsevier Science.

Pasaogullari, U. and Wang, C. Y. (2004). "Liquid Water Transport in Gas Diffusion Layer of Polymer Electrolyte Fuel Cells." Lournal of The Electrochemical Society 151(3): A 399-A 406.

Rashapov, R. R., Unno, J. and Gostick, J. T. (2015). "Characterization of PEM FC Gas Diffusion Layer Porosity." Lournal of The Electrochemical Society 162(6): F603-F612.

Rooks, M. A. M. M. J. (1997). SPIE Handbook of Microlithography, Micromachining and Microfabrication. 
Salvat F., F.-V. J. M . a. S. J. "PENEL OPE-2011: A Code System for M onte Carlo Simulation of Electron and Photon Transport." OECD NEA Data Bank/N SC DOC(2011)/5.

Sempau, J., A costa, E., Baro, J., Fernández-Varea, J. M . and Salvat, F. (1997). "A n al gorithm for M onte Carlo simulation of coupled electron-photon transport." Nuclear Instruments and M ethods in Physics Research Section B: B eam Interactions with M aterials and A toms 132(3): 377-390.

Sempau, J., Fernández-Varea, J. M., A costa, E. and Salvat, F. (2003). "Experimental benchmarks of the M onte Carlo code penelope." Nuclear Instruments and M ethods in Physics Research Section B: B eam Interactions with M aterials and A toms 207(2): 107-123.

Shore, K. A . (2013). "N anofabrication U sing Focused Ion and Electron B eams: Principles and Applications, edited by Ivo Utke, Stanisislav M oshkalev and Phillip Russell." Contemporary Physics 54(2): 140-141.

Stannett, V. T. (1990). "Radiation grafting - state-of-the-art." International Journal of Radiation A pplications and Instrumentation. Part C. Radiation Physics and Chemistry 35(1): 82-87.

Sun, Z. and Karppi, R. (1996). "The application of electron beam welding for the joining of dissimilar metals: an overview." Lournal of M aterials Processing Technology 59(3): 257-267.

Vieu, C., Carcenac, F., Pépin, A., Chen, Y., M ejias, M., Lebib, A., M anin-Ferlazzo, L., Couraud, L. and Launois, H. (2000). "Electron beam lithography: resolution limits and applications." A pplied Surface Science 164(1-4): 111-117.

Weber, A. Z. and Newman, J. (2005). "Effects of M icroporous Layers in Polymer Electrolyte Fuel Cells." Lournal of The Electrochemical Society 152(4): A 677-A 688.

Wolff-Fabris, F., A ltstädt, V., A rnold, U. and Döring, M . (2011). Examples of Electron B eam Curing A pplications. Electron B eam, Hanser: 105-114.

Zahner, D., A bagat, J., Svec, F., Fréchet, J. M . J . and L evkin, P. A . (2011). "A Facile A pproach to Superhydrophilic-Superhydrophobic Patterns in Porous Polymer Films." Advanced M aterials 23(27): 3030-3034. 